DOI: 10.1002/adsc.201400871

\title{
Opportunities in Photocatalytic Synthesis
}

\author{
Tehshik P. Yoon ${ }^{\mathrm{a}, *}$ and Corey R. J. Stephenson ${ }^{\mathrm{b}, *}$
}

a Department of Chemistry, University of Wisconsin - Madison, 1101 University Ave., Madison, WI 53706-1396, USA

Fax: (+1)-608-265-4534; e-mail: tyoon@chem.wisc.edu

b Department of Chemistry, University of Michigan, 930 N. University Ave., Ann Arbor, MI 48109, USA

Fax: (+1)-734-647-4865; e-mail: crjsteph@umich.edu

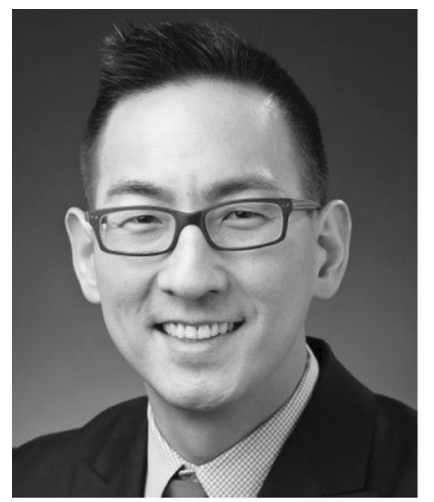

Tehshik P. Yoon

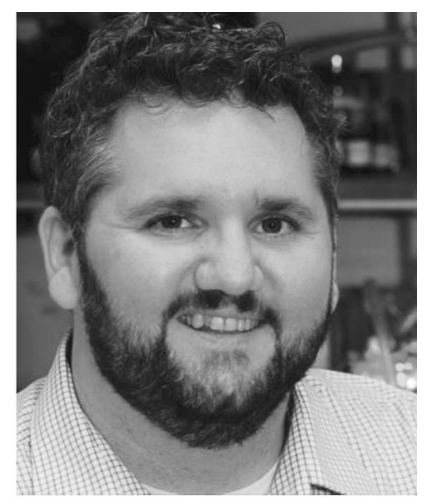

Corey R. J. Stephenson

Over the past several years, there has been a renewed appreciation for the synthetic capabilities afforded by photochemical activation. Chemists' fascination with light's ability to promote chemical changes in organic molecules dates back at least as far as Ciamician's pioneering studies in organic photochemistry at the turn of the $20^{\text {th }}$ century. This longstanding interest has been motivated by the distinctive chemical transformations that can uniquely be accomplished using photochemistry, by the exquisite spatial and temporal control that photochemistry provides, and the long-standing hope that the ability to use safe and renewable solar photons in the production of chemicals might improve the sustainability of synthetic processes.

While photochemistry is by no means a new concept in synthetic organic chemistry, our community's

interest in photochemical techniques has enjoyed tremendous growth in the past few years. Much of the recent research in photochemical synthesis has focused on the theme of photocatalysis, consistent with the importance that catalysis has had in all aspects of modern chemistry. In particular, the strategies that have been so productively exploited in the design of novel technologies for solar energy applications are now being shown to be equally powerful in the synthesis of complex organic structures.

The catalysts that have become commonly utilized in synthetic photocatalysis span a broad range of structures, including transition metal complexes, relatively simple organic chromophores, and chiral hydrogen bonding scaffolds. Mechanistically, the modes of action demonstrated by light-activated catalysts are equally diverse; reactions involving photoinduced electron transfer, energy transfer, and atom transfer have all proven to be the basis for powerful new synthetic methods described in the past few years.

Each of these varied approaches to photocatalytic synthesis can be found featured in this special issue of Advanced Synthesis \& Catalysis. Collectively, the papers in this issue provide an excellent demonstration of the many ways in which photocatalysis is having an impact in contemporary synthetic organic chemistry. We hope that this special issue will serve as a valuable entry point for chemists who would like to learn more about the array of opportunities presented by photochemical synthesis. 